\title{
DETERMINACIÓN DE LOS NIVELES DE COLESTEROL LDL EN UNA ESPECIE CON PATRÓN HDL
}

\author{
Measurement of LDL Cholesterol Levels in a Species with HDL Pattern
}

\author{
José Henry Osorio ${ }^{1}$
}

\section{Resumen}

Los métodos utilizados de manera rutinaria para la determinación del perfil lipídico en humanos, una especie con patrón metabólico LDL ("Lipoproteínas de baja densidad") pueden ser inadecuados para la determinación del perfil lipídico en especies con patrón HDL ("Lipoproteínas de alta densidad"). El presente estudio tuvo por objetivo comparar el método de precipitación y el método de Friedewald para la determinación del colesterol LDL en una especie con patrón HDL. Se tomaron muestras de sangre, en estado de ayuno, de 200 equinos de diferentes edades sin discriminación de sexo. Se extrajo el suero y se determinaron los niveles de colesterol LDL mediante los dos métodos en estudio. Los valores con el método de precipitación fueron de $32 \pm 8.8 \mathrm{mg} / \mathrm{dl}$ y por el método de Friedewald de $29 \pm 7.7 \mathrm{mg} / \mathrm{dl}(\mathrm{p}<0.05)$. Dada la diferencia de valores entre métodos, se recomienda el uso del método de Friedewald para determinar los niveles de colesterol LDL en el esquino, especie con patrón HDL.

Palabras clave: lípidos, colesterol LDL, equino

\section{AbSTRaCT}

The methods used for lipid profile measurement in humans, a species with LDL ("Low-density lipoprotein") pattern, can be unsuitable for the lipid profile determination in species with HDL ("High-density lipoprotein") pattern. The objective of this study was to compare the precipitation method and the Friedewald method for the measurement of LDL cholesterol in a species with HDL pattern. Blood samples were collected after $24 \mathrm{~h}$ fasting in 200 horses of different ages and without discrimination by sex. After serum extraction, levels of LDL cholesterol were determined

\footnotetext{
${ }^{1}$ Laboratorio de Investigación en Bioquímica Clínica y Patología Molecular, Departamento de Ciencias Básicas de la Salud, Universidad de Caldas. E-mail: Jose.osorio_o@ucaldas.edu.co
}

Recibido: 13 de diciembre de 2012

Aceptado para publicación: 16 de mayo de 2013 
by both methods. The values obtained by the precipitation method were $32 \pm 8.8 \mathrm{mg} / \mathrm{dl}$ and for the Friedewald method were $29 \pm 7.7 \mathrm{mg} / \mathrm{dl}(\mathrm{p}<0.05)$. Given the difference in values between methods, it is recommended the Friedewald method for determining levels of LDL cholesterol in the horse, a species with HDL pattern.

Key words: lipids, HDL cholesterol, equine

\section{INTRODUCCIÓN}

El transporte de lípidos que provienen de la dieta, así como de los que se forman en el hígado, se realiza mediante las lipoproteínas. El metabolismo de las lipoproteínas difiere en cada especie, pero algunas tienen características muy similares. Los conejos, cobayos, camélidos, hámster, rinocerontes, cerdos y la mayor parte de los monos, muestran predominancia de lipoproteínas de baja densidad (LDL), al igual que en el humano (Tebot, 2000). En estas especies, un aumento de grasa y colesterol en la dieta se refleja rápidamente en un aumento de LDL. Por otro lado, en el caso de equinos, rumiantes, ratas, felinos y caninos predominan las lipoproteínas de alta densidad (HDL) y muestran aumentos de HDL como respuesta al aumento de grasas y colesterol en la dieta (Bauer, 1997).

Los métodos utilizados de manera rutinaria para la determinación del perfil lipídico en humanos, una especie con patrón LDL, pueden ser inadecuados para la determinación del perfil lipídico en especies con patrón HDL, debido al diferente metabolismo lipídico (Coppo et al., 2003).

Dentro de los métodos propuestos para la determinación del perfil lipídico, el más ampliamente utilizado es el de Friedewald (Friedewald et al., 1972). Este método se utiliza rutinariamente en los laboratorios de bioquímica clínica humana y en algunos de bioquímica clínica veterinaria; sin embargo, se requiere saber si existe justificación para usar este método en animales que presentan un patrón metabólico diferente al del humano. El método de Friedewald no utiliza mediciones directas del colesterol LDL, siendo este valor calculado en base a otros parámetros.

Hay métodos que determinan este parámetro. Así se tiene el método directo, el cual emplea un detergente específico que solubiliza el colesterol de las lipoproteínas HDL, VLDL (de muy baja densidad) y quilomicrones. Los ésteres de colesterol son hidrolizados por la colesterol esterasa y la colesterol oxidasa mediante una reacción no formadora de color, donde después, un segundo detergente solubiliza el colesterol de las lipoproteínas LDL de la muestra para ser cuantificado por espectrofotometría (Tiez, 2006; Young, 1995). No obstante estas ventajas, es un método costoso. Otro método es el de precipitación (Assmann et al., 1984), método de bajo coste, que permite hacer mediciones directas del colesterol LDL, pero que puede presentar interferencias debido a las concentraciones de los reactivos (Nauck et al., 2002).

El presente trabajo tuvo como objetivo comparar el método de precipitación con el método de Friedewald (utilizado de manera rutinaria para la determinación del colesterol LDL en humanos) para la determinación del colesterol LDL en una especie con patrón metabólico HDL. 


\section{Materiales y Métodos}

Se trabajó con 200 equinos de diversa edad y sin discriminación de sexo, del departamento de Caldas, Colombia. Los animales eran criados bajo un sistema de estabulación y con un plan sanitario adecuado (vacunas y desparasitaciones al día). La condición corporal variaba entre 4 a 5 , medida en una escala de 1 a 9 .

Los animales se dejaron en estado de ayuno para la toma de la muestra. Se recolectaron $5 \mathrm{ml}$ de sangre de cada animal y las muestras fueron centrifugadas a $3500 \mathrm{rpm}$ durante 15 minutos. El suero fue extraído para determinar los niveles de colesterol LDL utilizando un kit comercial (BioSystems ${ }^{\circledR}$ ) mediante el método de precipitación (Assmann et al., 1984), donde las LDL precipitan en presencia de sulfato polivinil. La concentración de colesterol LDL se calcula por diferencia entre los valores de colesterol total medido espectrofotométricamente en el suero y los valores de colesterol determinados en el sobrenadante obtenido tras la precipitación.

Posteriormente, se determinaron los niveles de colesterol LDL mediante el método de Friedewald (Friedewald et al., 1972) cuyo fundamento es el siguiente: el colesterol HDL es precipitado en presencia de ácido fosfotúngstico y determinado mediante el método enzimático-colorimétrico aplicado para la determinación del colesterol total de la muestra. Además, se determina el nivel de los triglicéridos mediante un método enzimático-colorimétrico. Los valores de los triglicéridos se dividen entre cinco, correspondiendo este valor a los niveles de colesterol-VLDL. Los valores de colesterol LDL fueron calculados mediante la siguiente fórmula: Colesterol LDL = Colesterol total - (colesterol HDL - colesterol VLDL).

Los resultados fueron analizados estadísticamente mediante el análisis de varianza de una vía y se hizo un análisis de correlación entre los valores resultantes de ambos métodos, utilizando el programa StatGraphics Centurium 15.14 (XV).

\section{Resultados}

Con el método de precipitación se obtuvo los valores promedio $( \pm \mathrm{DE})$ de $32 \pm 8.8$ $\mathrm{mg} / \mathrm{dl}$ y valores mínimo y máximo de 13 y 59 $\mathrm{mg} / \mathrm{dl}$, respectivamente, en tanto que con el método de Friedewald se obtuvo valores promedio de $29 \pm 7.0 \mathrm{mg} / \mathrm{dl}$ y valores mínimo y máximo de 11.2 y $54.2 \mathrm{mg} / \mathrm{dl}$, respectivamente, habiendo diferencia estadística entre los resultados de ambos métodos analíticos $(\mathrm{p}<0.05)$. Asimismo, la correlación entre los dos métodos fue positiva $(\mathrm{r}=0.54)$ aunque no significativa (Fig. 1).

\section{Discusión}

De acuerdo al metabolismo lipídico, las especies se incluyen dentro de dos grandes patrones con base en el tipo de lipoproteína encargada del transporte del colesterol al interior del organismo (Osorio y Giraldo, 1999). El patrón LDL (LDL>HDL) se caracteriza por altos niveles de C-LDL, por lo que exhiben mayor riesgo aterogénico, y el patrón HDL (HDL>LDL) donde la mayor parte del colesterol es transportado por las lipoproteínas de alta densidad, y estos individuos tienen menor riesgo de presentar aterogénesis (Osorio et al., 2008).

El perfil lipídico incluye la determinación en suero sanguíneo de los niveles de colesterol total (CT), colesterol HDL (C-HDL), colesterol VLDL (C-VLDL), colesterol LDL (C-LDL) y triglicéridos, el cual puede estar alterado en casos de hipotiroidismo, diabetes mellitus, obesidad, síndrome nefrótico, pancreatitis aguda, ictericia obstructiva, hiperadrenocorticismo, ciertas retinopatías, insuficiencia hepática, síndrome de mala absorción, errores innatos del metabolismo e hipertiroidismo, entre otros (NCEP, 2001a). 


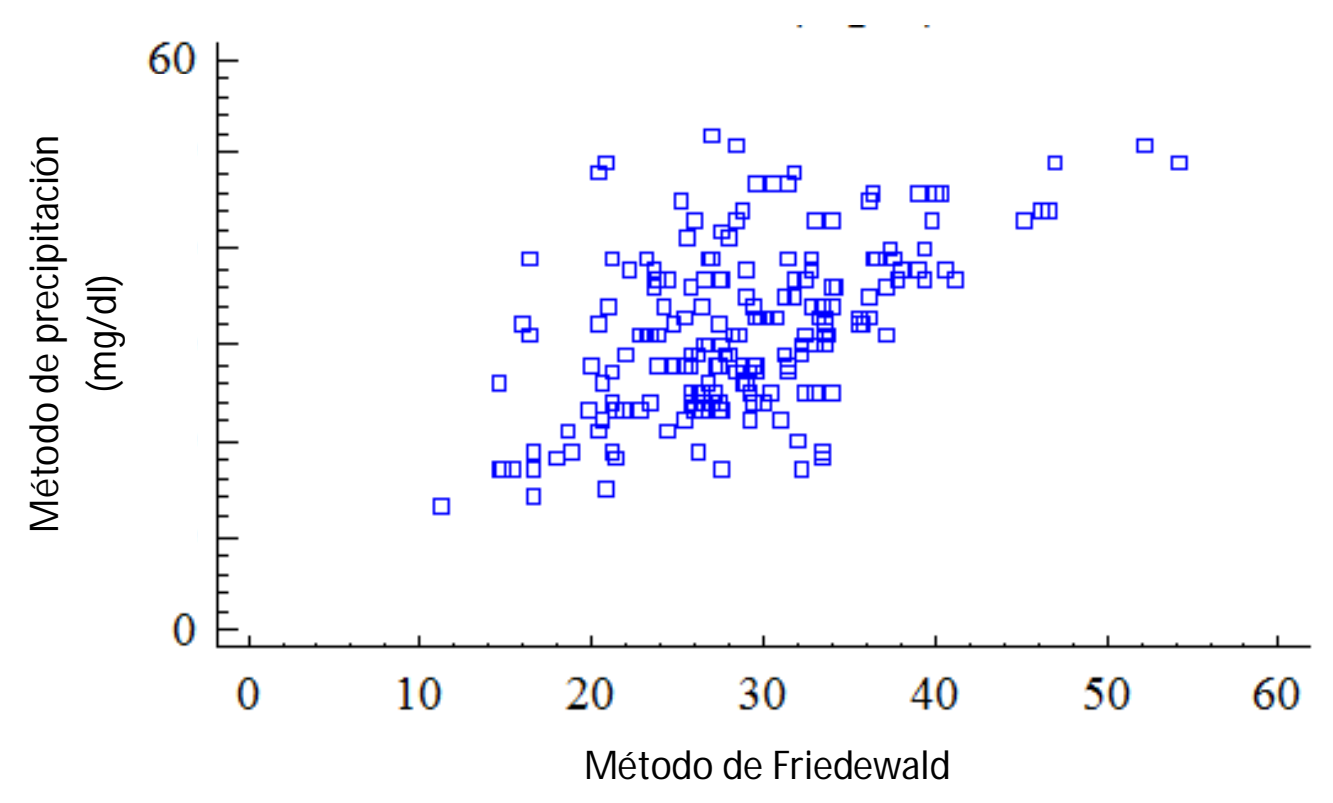

$(\mathrm{mg} / \mathrm{dl})$

Figura 1. Comparación de los valores de colesterol-LDL en una especie con patrón HDL determinados por el método de precipitación versus el método de Friedewald $(r=0.54)$

No existen referencias relacionadas con la determinación del perfil lipídico completo en especies con patrón HDL. En el estudio de Coppo et al. (2003), se evaluó el colesterol ligado a lipoproteínas de alta y baja densidad (C-HDL y C-LDL) en varias especies de animales domésticos mediante la técnica enzimática de oxidasa-peroxidasa. En el equino se reportaron valores de colesterol LDL de $30 \pm 16 \mathrm{mg} / \mathrm{dl}$, siendo valores intermedios a los obtenidos en el presente estudio con los dos métodos evaluados (Precipitación: $32 \pm$ $8.8 \mathrm{mg} / \mathrm{dl}$; Friedewald: $29 \pm 7.0 \mathrm{mg} / \mathrm{dl}$ ). Asimismo, en un estudio en equinos, Osorio y Uribe (2011) determinaron los valores de colesterol LDL por el método directo y la formula de Friedewald sin encontrar diferencia entre ambos métodos.

Algunos investigadores, trabajando con especies HDL, han encontrado diferencias en los niveles de colesterol total por efecto de la dieta (Coles, 1989), edad (Groslambert et al., 1985) o raza (Downs et al., 1993). Asimismo, es necesario hacer énfasis acerca del estilo de vida de los animales en sus respectivos ambientes; sin embargo, los valores reportados muestran a veces grandes diferencias por factores adicionales. Por ejemplo, se han descrito severos trastornos del metabolismo lipídico, como la hiperlipemia de los ponies Shetland, cuya patogenia es confusa, apareciendo como un trastorno secundario a enfermedades preexistentes o en forma espontánea como resultado de una privación de alimentos (Bruss, 2008).

Los métodos de laboratorio usados son importantes al evaluar cuales pueden ser los valores de perfil lipídico en especies con patrón HDL. En humanos, una especie LDL, el método de Friedewald es ampliamente utilizado en los laboratorios clínicos, considerándose como el método de referencia, ya que la ß-cuantificación por ultracentrifugación no es adecuada para uso rutinario (NCEP, 
2001b). Sin embargo, la ecuación ha sido cuestionada, particularmente desde el concepto que la mayoría de los triglicéridos residen en la fracción VLDL y de que la relación entre los triglicéridos y el colesterol en esta fracción es constante (Warnick et al., 1990). Se han introducido ensayos homogéneos para determinar colesterol LDL, pero aún necesitan validación adicional (Nauck et al., 2002).

Otras vías alternativas han sido propuestas para determinar colesterol LDL. Una de ellas tiene en cuenta la concentración total de apolipoproteína B (apo-B). Así, la ecuación $\mathrm{LDL}-\mathrm{C}=0.41 \mathrm{TC}-0.14 \mathrm{TG}+0.66 \mathrm{apoB}$ - 10.43 ha sido propuesta para estimar colesterol LDL (Planella et al., 1997) y las ecuaciones LDL-C $=0.94 \mathrm{TC}-0.94 \mathrm{HDL}-\mathrm{C}$ $-0.19 \mathrm{TG}$ y LDL-apoB $=$ apoB $-0.09 \mathrm{TC}+$ 0.09HDL-C - 0.08TG han sido propuestas para la determinación de colesterol LDL-C y apoB en las LDL y, por lo tanto, el cálculo de la relación LDL-C/LDL-apoB (Hattori et al., 1998). Estas relaciones buscan determinar el tamaño de las partículas LDL.

El presente trabajo, al analizar la conveniencia de la utilización del método de rutina (Friedewald) para la determinación de LDL y el método de precipitación encontró que los dos métodos muestran resultados estadísticamente diferentes $(\mathrm{p}<0.05)$. Es así, que teniendo en cuenta los pocos estudios realizados y las interferencias que se pueden encontrar en los métodos de precipitación, se recomienda la utilización del método de Friedewald para la medición de colesterol LDL en especies con patrón HDL; sin embargo, se recomienda asimismo, investigaciones adicionales.

\section{Agradecimientos}

El autor hace constar su agradecimiento a María Elena Álvarez por su colaboración en el trabajo de laboratorio y a Jazmín Vinazco por la colaboración en la corrección del manuscrito.

\section{Literatura Citada}

1. Assmann G, Jabs HU, Kohnert U, Nolte W, Schriewer H. 1984. LDLcholesterol determination in blood serum following precipitation of LDL with polyvinylsulfate. Clin Chim Acta 140: 77-83.

2. Bauer JE. 1997. Metabolismo comparado de lípidos y lipoproteínas. Pet's Ciencia 13: 362-376.

3. Bruss ML. 2008. Lipids and ketones. In: Kaneko JJ, Harvey JW, Bruss ML (eds). Clinical biochemistry of domestic animals. $6^{\text {th }}$ ed. San Diego, USA: Academic Press/Elsevier. p 81-117.

4. Coles EH. 1989. Veterinary clinical pathology. $4^{\text {th }}$ ed. Philadelphia: Saunders. $486 \mathrm{p}$.

5. Coppo NB, Coppo JA, Lazarte MA. 2003. Intervalos de confianza para colesterol ligado a lipoproteínas de alta y baja densidad en suero de bovinos, equinos, porcinos y caninos. Rev Vet 14: 1-10.

6. Downs L, Bolton CH, Crispin S. 1993. Plasma lipoprotein lipids in five different breeds of dogs. Res Vet Sci 54: 63-67.

7. Friedewald WT, Levy RI, Fredrickson DS. 1972. Estimation of the concentration of low-density lipoprotein cholesterol in plasma, without use of the preparative ultracentrifuge. Clin Chem 18: 499-502.

8. Groslambert P, Foulon T, Groulade J, Groulade P. 1985. Lipid and lipoproteins in the normal dog in relation to age and sex. B Acad Vet France 58: 473-484.

9. Hattori Y, Suzuki M, Tsushima M, Yoshida M, Tokunaga Y, Wang Y, Zhao D, et al. 1998. Development of approximate formula for LDL-Chol, LDL-apo B and LDL-Chol/LDL-apoB as indices of hyperapobetalipoproteinemia and small dense LDL. Atherosclerosis 138: 289-299.

10. Nauck M, Warnick GR, Rifai N. 2002. Methods for measurement of LDLCholesterol: a critical assessment of 
direct measurement by homogeneous assays versus calculation. Clin Chem 48 : 236-254.

11. [NCEP] National Cholesterol Education Program Expert Panel. 2001a. Third report of the National Cholesterol Education Program (NCEP) Expert Panel on Detection, Evaluation, and Treatment of High Blood Cholesterol in Adults (ATP III). Bethesda: National Heart, Lung, and Blood Institute. $284 \mathrm{p}$.

12. [NCEP]. National Cholesterol Education Program Expert Panel. 2001b. Executive summary of the third report of The National Cholesterol Education Program (NCEP) Expert Panel on Detection, Evaluation, and Treatment of High Blood Cholesterol in Adults (Adult Treatment Panel III). JAMA 285: 2486-2497.

13. Osorio JH, Giraldo CE. 1999. Perfil lipídico en caninos adultos obesos versus perfil lipídico en caninos adultos normales. Rev Vet Zoot 11: 22-27.

14. Osorio JH, Loango-Chamorro N, Landázuri P. 2008. El proceso aterosclerótico desde el periodo fetal a la edad adulta basado en el transporte del colesterol endógeno. Rev Asoc Col Cienc Biol 20: 173-191;

15. Osorio J, Uribe L. 2011. Comparación de los métodos Directo y de Friedewald para la determinación de los niveles de colesterol LDL en el equino. Rev MVZ Córdoba 16: 2549-2553.

16. Planella T, Cortes M, Martinez-Bru C, Gonzalez-Sastre F, Ordonez-Llanos J. 1997. Calculation of LDL-Cholesterol by using apolipoprotein B for classification of nonchylomicronemic dyslipemia. Clin Chem 43: 808-815.

17. Tebot CA. 2000. Fisiología metabólica de los rumiantes. Montevideo: EdCSIC. $341 \mathrm{p}$.

18. Tietz WA. 2006. Clinical guide to laboratory tests. $4^{\text {th }}$ ed. New York: Saunders/Elsevier. 1798 p.

19. Warnick GR, Knopp RH, Fitzpatrick V, Branson L. 1990. Estimating low density lipoprotein cholesterol by the Friedewald equation is adequate for classifying patients on the basis of nationally recommended cutpoints. Clin Chem 36: 15-19.

20. Young DS. 1995. Effects of drugs on clinical laboratory tests. $4^{\text {th }}$ ed. Washington DC, USA: AACC Press. $161 \mathrm{p}$. 